\title{
New Challenges in Nuclear Endocrinology
}

\author{
David Taïeb ${ }^{1}$, Rodney J. Hicks² , and Karel Pacak ${ }^{3}$ \\ ${ }^{I}$ Department of Nuclear Medicine, La Timone University Hospital, CERIMED, Aix-Marseille University, Marseille, France; ${ }^{2}$ Cancer \\ Imaging and Neuroendocrine Tumour Service, Peter MacCallum Cancer Centre, and Sir Peter MacCallum Department of Oncology, \\ University of Melbourne, Melbourne, Australia; and ${ }^{3}$ Section on Medical Neuroendocrinology, Eunice Kennedy Shriver National \\ Institute of Child Health and Human Development, National Institutes of Health, Bethesda, Maryland
}

$\mathbf{M}$ integration of clinical observations, and diagnostic test results. It is without any doubt that this comprehensive approach to a patient, based on rigorous evaluation of different data, originally termed the anatomic-clinical method, has proven to be the most effective methodology for understanding human diseases.

In recent years, genomics has profoundly informed the management of certain diseases. Progressively, genetic analysis and molecular pathologic testing for aberrant gene products have been integrated into patient management, influencing therapeutic choices, follow-up strategies, and the surveillance and counseling of family members. This comprehensive approach has been termed precision medicine and also considers the role of environmental factors. Recently, rapidly evolving understanding of somatic mutations, particularly the role of gene variants in a disease pathogenesis, epigenetic events, and analysis of big data in the context of genomic profiles, has augmented our understanding of disease by providing new insights into genome regulation-exciting explanations for different phenotypes that can be observed across the same genotypes. This new insight has also challenged our simplistic assumption that the genetic status of patients and their tumors is sufficient to guide individualized therapy. In this evolving situation, the following question arises: How can we understand and integrate such complex data in the optimization of patient care? Despite progress made in "omics" profiling and bioinformatics, a significant portion of useful data is still escaping our radars. Then, could molecular imaging, by virtue of phenotyping disease, be another important connecting hub between disease characterization and precision medicine? In fact, every imaging phenotype could be viewed as a molecular biomarker that reflects and integrates a wide variety of pathophysiologic events, including genetic or epigenetic alterations.

Endocrinology has a long tradition of use in molecular imaging for detection and characterization of disease - a tradition built on the seminal work in thyroid disorders performed in the last century. The opportunity to build on this experience is apparent because of the diversity of molecular targets, including hormone

Received Oct. 22, 2017; revision accepted Oct. 30, 2017.

For correspondence or reprints contact: David Taïeb, Service de Médecine Nucléaire, Centre hospitalo-universitaire de la Timone, Centre Européen de Recherche en Imagerie Médicale, Université Aix-Marseille, 264, rue SaintPierre, 13385 Marseille, France.

E-mail: david.taieb@ap-hm.fr

Published online Nov. 9, 2017.

COPYRIGHT (c) 2018 by the Society of Nuclear Medicine and Molecular Imaging. DOI: 10.2967/jnumed.117.199729 synthesis pathways expressed uniquely by endocrine tumors, and the growing knowledge of their pathophysiology and genetics.

What are the prospects for nuclear endocrinology? Our concept of this future can be described by what we call the PTI approachprognostication and tumor grading $(\mathrm{P})$, therapeutic drug monitoring (T), and immune system (I):

Prognostication and Tumor Grading. Several studies have shown that ${ }^{18} \mathrm{~F}$-FDG provides valuable prognostic information for certain tumors such as thyroid cancers (medullary and follicular) and neuroendocrine tumors (NETs) (1-4). Its prognostic role, alongside histopathologic data (tumor histology, Ki-67), tumor growth evolution, and biomarker kinetics enables a better stratification of patients and influences treatment decisions. The next step would be to incorporate ${ }^{18} \mathrm{~F}$-FDG phenotypes in the classification of these tumors (e.g., refractory thyroid cancers, NET grading system). At present, somatostatin receptor-based imaging remains the optimal noninvasive approach to determine whether a NET (e.g., bronchial and gastroenteropancreatic NETs) expresses differentiated features in the same way that radioactive iodine uptake is used to define the differentiated nature of follicular thyroid cancer. The progressive loss of somatostatin receptor expression and increase in ${ }^{18} \mathrm{~F}$-FDG avidity as manifestations of dedifferentiation of neuroendocrine neoplasia have been shown to have significant prognostic implications (2,3), and the presence of spatial discordance in the distribution of these molecular imaging phenotypes has implications for the use of peptide receptor radionuclide therapy (4). Interestingly, pheochromocytomas and paragangliomas are an exception, as these less differentiated tumors ( $\mathrm{SDHx}$ mutated) strongly express somatostatin, opening the door for unprecedented therapeutic approaches (5). Furthermore, high uptake of ${ }^{18} \mathrm{~F}$-FDG in these tumors is a manifestation of metabolic reprogramming rather than dedifferentiation or aggressivity and therefore not necessarily an indicator of adverse prognosis as it is in other types of neoplasia (6).

Therapeutic Drug Monitoring. It has recently been shown that beyond ${ }^{18} \mathrm{~F}$-FDG uptake by tumor cells, ${ }^{18} \mathrm{~F}$-FDG phenotypes can reflect tumor-stroma interactions (7). For example, pheochromocytomas and paragangliomas related to mutations in the genes encoding for the succinate dehydrogenase are associated with extremely high accumulation of succinate, an oncometabolite that is capable of interacting within tumor cells and also with the surrounding stroma. Therefore, ${ }^{18} \mathrm{~F}$-FDG uptake can also be viewed in certain malignancies as a marker of endothelial metabolic activity, which could be used to monitor a response to antiangiogenic therapy. ${ }^{18}$ F-FDG PET is extensively applied in oncology; its use for phenotyping tumor-stroma interactions should not be limited 
to pheochromocytomas and paragangliomas and merits evaluation in other endocrine malignancies by interrogating metabolic and metabolomics data. Phenotyping of $6{ }^{18} \mathrm{~F}$-fluoro-L-dopa is more complex than it seems; even though $6-{ }^{18} \mathrm{~F}$-fluoro-L-dopa uptake is the commonest finding in NETs, it remains dependent on nonspecific phenomena (large neutral amino acid transporter). However, tracer retention depends on differentiation profiles such as catecholaminergic and serotoninergic phenotypes (8). Therefore, $6-{ }^{18}$ F-fluoro-L-dopa provides information on tumor differentiation for certain NET tumors. When considering other tumors, one should also view it as a "nutrient" radiopharmaceutical like ${ }^{18} \mathrm{~F}$ FDG. Perhaps the evaluation of tumor response by different nutrient tracers could be used in, for example, pancreatic NETs. This is not a trivial example, since amino acid nutrition in mammalian cells is coupled to cell signaling via the mammalian target of rapamycin.

Immune System. Recently, immunotherapy with immune checkpoint inhibitors has revolutionized the therapeutic approach to several malignancies. Although this strategy is currently under investigation in endocrine malignancies, it is expected that tumors with a higher genetic instability (anaplastic and poorly differentiated thyroid cancer, G3 gastroenteropancreatic NETs, and adrenocortical carcinoma) would be the best responders to this approach (9). In cases of low mutational load, radiation may be clinically beneficial by inducing de novo antitumor immune responses. Therapeutic nuclear medicine could also be an efficient synergistic combination. In these patients, we are yet to discover noninvasive imaging of immune ligands in tumors, metastases for the evaluation of neoantigen landscape dynamics, and the optimal temporal sequencing of different approaches.

We feel that the PTI approach further justifies the rationale for nuclear endocrinology to be well implemented in current clinical practice. We predict that with improved molecular biology methods, proteomics, and metabolomics, together with application of sophisticated bioinformatics algorithms and molecular biologic approaches (RNA sequencing, detection of cell membrane targets by proteomic analyses, metabolomics, and big data analysis and storage), new radiopharmaceuticals will soon be introduced (10). Such radiopharmaceuticals are expected to be highly specific for NETs, which uniquely, in comparison to other tumors, possess specific hormone synthesis and signaling pathways, hormonerelated cytoplasmic and cell membrane receptor transporters, specific metabolomic pathways regulated by hormones themselves, and epigenetic events induced by the presence of various hormones. Those radiopharmaceuticals not only will be used in specific detection of a particular endocrine disorder or tumor but also will serve as a platform for developing new radiotherapeutics and other treatment strategies. They will also be paramount in the prediction of some therapeutic options that are to be used in a particular patient to monitor intracellular events (e.g., degree of hypoxia, apoptosis, proliferation, and immune processes) that are induced by various therapies. Finally, they may be able to predict the aggressiveness and metastatic potential of these tumors. The PTI approach, by complementing our deep knowledge and future discoveries about the pathogenesis of these tumors, together with the introduction of new radiopharmaceuticals, will further strengthen our dream of personalized medicine that will indeed be able to reach and benefit a patient.

\section{DISCLOSURE}

No potential conflict of interest relevant to this article was reported.

\section{REFERENCES}

1. Ezziddin S, Adler L, Sabet A, et al. Prognostic stratification of metastatic gastroenteropancreatic neuroendocrine neoplasms by ${ }^{18} \mathrm{~F}-\mathrm{FDG}$ PET: feasibility of a metabolic grading system. J Nucl Med. 2014;55:1260-1266.

2. Garin E, Le Jeune F, Devillers A, et al. Predictive value of ${ }^{18}$ F-FDG PET and somatostatin receptor scintigraphy in patients with metastatic endocrine tumors. J Nucl Med. 2009;50:858-864.

3. Binderup T, Knigge U, Loft A, Federspiel B, Kjaer A. ${ }^{18}$ F-fluorodeoxyglucose positron emission tomography predicts survival of patients with neuroendocrine tumors. Clin Cancer Res. 2010;16:978-985.

4. Hicks RJ. Use of molecular targeted agents for the diagnosis, staging and therapy of neuroendocrine malignancy. Cancer Imaging. 2010;10(spec no A):S83-S91.

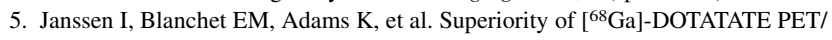
CT to other functional imaging modalities in the localization of SDHB-associated metastatic pheochromocytoma and paraganglioma. Clin Cancer Res. 2015;21: 3888-3895.

6. Jochmanova I, Pacak K. Pheochromocytoma: the first metabolic endocrine cancer. Clin Cancer Res. 2016;22:5001-5011.

7. Garrigue P, Bodin-Hullin A, Balasse L, et al. The evolving role of succinate in tumor metabolism: an ${ }^{18}$ F-FDG-based study. J Nucl Med. 2017;58:1749-1755.

8. Deroose CM, Hindie E, Kebebew E, et al. Molecular imaging of gastroenteropancreatic neuroendocrine tumors: current status and future directions. $J \mathrm{Nucl}$ Med. 2016;57:1949-1956.

9. Latteyer S, Tiedje V, Schilling B, Fuhrer D. Perspectives for immunotherapy in endocrine cancer. Endocr Relat Cancer. 2016;23:R469-R484.

10. Crona J, Taieb D, Pacak K. New perspectives on pheochromocytoma and paraganglioma: towards a molecular classification. Endocr Rev. 2017;38:489-515. 